\title{
PAPER
}

\section{Recanalisation of cerebral venous thrombosis}

\section{R W Baumgartner, A Studer, M Arnold, D Georgiadis}

See end of article for

authors' affiliations

J Neurol Neurosurg Psychiatry 2003;74:459-461

.....................

Correspondence to: Dr Ralf W Baumgartner Department of Neurology, University Hospital, Fravenklinikstr 26, $\mathrm{CH}-8091$ Zürich, Switzerland; ralf.baumgartner@nos.usz.ch

Received 11 September 2002

In revised form

13 November 2002

Accepted

11 December 2002
Objective: To investigate recanalisation in the first 12 months after cerebral venous thrombosis. Methods: 33 consecutive patients presenting with cerebral venous thrombosis were enrolled in the study. Diagnosis was made by magnetic resonance imaging (MRI) and magnetic resonance venography (MRV) or catheter angiography. Patients were initially treated with intravenous heparin. Warfarin was given for at least four months. Cerebral MRI and MRV were done at four months and repeated after 12 months if venous thrombosis persisted. Outcome was evaluated by the Rankin scale at 12 months. Results: Outcome at 12 months was good, with a median modified Rankin scale score of 0 (range 0 to 2$) ; 27$ patients (82\%) had no residual deficits. No patient suffered recurrent cerebral venous thrombosis, deep vein thrombosis, or pulmonary embolism during follow up. After four months, all deep cerebral veins and cavernous sinuses, $94 \%$ of superior sagittal sinuses, $80 \%$ of straight sinuses, $73 \%$ of jugular veins, $58 \%$ of transverse sinuses, and $41 \%$ of sigmoid sinuses had recanalised. No further recanalisation was observed thereafter.

Conclusions: The results suggest that recanalisation only occurs within the first four months following cerebral venous thrombosis and not thereafter, irrespective of oral anticoagulation.
C erebral venous thrombosis is an infrequent disease that mainly affects young to middle aged patients. ${ }^{12}$ Its natural course varies from complete recovery to severe neurological deficit or death. ${ }^{1-3}$ Most clinicians recommend the administration of heparin early in the course of the disease, because this has been shown to be moderately beneficial compared with placebo. ${ }^{13}$ There is no consensus about the duration of subsequent oral anticoagulant treatment.

Digital subtraction angiography (DSA) is still the standard of reference for the assessment of cerebral venous thrombosis. ${ }^{4}$ However, combined magnetic resonance imaging (MRI) and magnetic resonance venography (MRV) have replaced carotid angiography in diagnostic work up of patients with a suspicion of cerebral venous thrombosis because these techniques are non-invasive and reliable, and also provide information about the brain parenchyma. ${ }^{4}$

Our aim in the present study was to investigate the recanalisation of cerebral venous thrombosis after four and 12 months of follow up, using cranial MRI and MRV.

\section{METHODS}

Consecutive patients with cerebral venous thrombosis diagnosed by MRI and MRV or DSA, without a previous history of neurological disease, were prospectively included in the study. All patients underwent brain imaging by computed tomography (CT) or MRI. Every patient was treated with intravenous heparin, followed by oral anticoagulation until the four month follow up. The duration of subsequent anticoagulation was at the discretion of the treating physician. Target international normalised ratio was 2.5 (range 2 to 3 ) for all patients and for the complete duration of warfarin treatment.

At the four months follow up all patients were examined by MRI and MRV. At the 12 months follow up clinical outcome was assessed by the modified Rankin scale. ${ }^{5}$ MRI and MRV were only done at 12 months in patients who had residual thrombosis of cerebral sinuses and veins at the four months follow up.

The study was conducted according to local ethics committee standards and the data were analysed without patient identification.

\section{Cerebral MRI, MRV, and carotid angiography}

Cerebral MRI and cross sectional time of flight (TOF) MRV studies were done on 1.5T magnetic resonance scanners. Section thickness of cross sectional TOF MRV was 1.5 to $2 \mathrm{~mm}$. Selective intra-arterial digital subtraction angiography was undertaken by a femoral artery approach in both internal carotid arteries in seven patients, in both vertebral arteries in six patients, and in one vertebral artery in one patient. Magnetic resonance and carotid angiography studies were reviewed retrospectively and independently at separate reading sessions by two of the authors, who were not aware of the ultrasound or clinical findings. Each cerebral vein or sinus was graded as open or occluded.

\section{Statistics}

Lilliefors testing ${ }^{6}$ indicated that the magnetic resonance variables were not normally distributed at presentation. Thus changes from values assessed at presentation were compared by non-parametric analysis of variance according to Friedman, followed by post hoc testing according to Wilcoxon and Wilcox in the case of overall significance. We used $\chi^{2}$ tests for comparison of frequencies. Probability $(p)$ values of less than 0.05 were considered significant.

\section{RESULTS}

Patients and neuroimaging studies

We enrolled 33 patients ( 22 women, 11 men), mean (SD) age 43 (15) years (range 21 to 77), with cerebral venous thrombosis diagnosed by MRI and MRV $(\mathrm{n}=26)$ or by carotid angiography $(n=7)$. Brain parenchyma was assessed by MRI in eight cases, by CT in two, and by both CT and MRI in 23.

\section{Neurological findings at presentation and after 12 months}

The results are given in table 1 . The most frequent neurological signs and symptoms were headache followed by focal signs, epileptic seizures, and impaired consciousness. After 12 months, 27 patients $(82 \%)$ had no residual deficits, and the median modified Rankin scale score was 0 (range 0 to 2 ). Residual deficits consisted of aphasia in four cases and motor hemiparesis in three. No patient suffered recurrent cerebral venous thrombosis, deep vein thrombosis, or pulmonary 
Table 1 Neurological findings at presentation and after 12 months in 33 patients with cerebral venous thrombosis

\begin{tabular}{lll}
\hline & $\begin{array}{l}\text { At presentation (n } \\
(\%))\end{array}$ & $\begin{array}{l}\text { At 12 months (n } \\
(\%))\end{array}$ \\
\hline Headache & $32(97)$ & 0 \\
Consciousness impaired & $10(32)$ & 0 \\
Confusion & $4(13)$ & 0 \\
Somnolence & $5(16)$ & 0 \\
Coma & $1(3)$ & 0 \\
Focal sign & $17(52)$ & $6(18)$ \\
Aphasia & $5(15)$ & $4(12)$ \\
Sensory deficit & $10(30)$ & 0 \\
Motor deficit & $9(27)$ & $3(9)$ \\
Hemianopia & $1(3)$ & 0 \\
Cranial nerve palsy & $1(3)$ & 0 \\
Epileptic seizure & $15(45)$ & 0 \\
\hline
\end{tabular}

embolism during follow up. No further epileptic seizures were observed after discharge.

The median duration of oral anticoagulation was 12 months (range 4 to 12). Twenty one patients were anticoagulated for 12 months, one for nine months, four for six to eight months, and seven for four to five months. The median duration of antiepileptic treatment was six months (range one to eight).

\section{Sites of thrombosis at presentation and at four and 12 months on MRV and carotid angiography}

These results are shown in table 2 . In 15 patients, venous recanalisation was incomplete after four months and also after 12 months. The length of warfarin administration was similar in patients with complete recanalisation (mean (SD), 11 (3) months; median 12, range five to 12) and in those with incomplete recanalisation (mean (SD), 9 (3); median 12; range four to 12$)(\mathrm{p}=0.2)$.

The transverse sinus, sigmoid sinus, and superior sagittal sinus were most frequently thrombosed $(85 \%, 73 \%$, and $48 \%$, respectively; table 2), whereas no deep middle cerebral veins or basilar veins were obstructed. After four months all deep cerebral veins and cavernous sinuses, 94\% of superior sagittal sinuses, $80 \%$ of straight sinuses, $73 \%$ of jugular veins, $58 \%$ of transverse sinuses, and $41 \%$ of sigmoid sinuses had recanalised. The differences in recanalisation rate were significant when patients with thrombosis of the transverse sinus, superior sagittal sinus, straight sinus, sigmoid sinus, and jugular vein were compared ( $p<0.001, \chi^{2}$ test), with superior sagittal sinus and straight sinus thromboses recanalising significantly more often than transverse sinus and sigmoid sinus thromboses (both $\mathrm{p}<0.05$ ). No further recanalisation was observed after the four month follow up.

\section{DISCUSSION}

The optimal long term treatment of cerebral venous thrombosis remains unclear, as no prospective randomised trials have yet examined this issue. Some clinicians adhere to therapeutic regimens used for the treatment of deep venous thrombosis. This strategy may be inappropriate because mechanisms underlying cerebral venous thrombosis and deep venous thrombosis are probably different. First, cerebral veins and sinuses have a continuous blood flow and no valves, ${ }^{4}$ whereas venous flow in the limbs is not continuous but requires the presence of valves and the activity of muscles. Second, cerebral venous thrombosis is not precipitated by situations involving immobilisation, such as surgery and travel, and may less often be caused by known cancer than deep venous thrombosis. $^{12}$ 7-10 Furthermore, even assuming that oral anticoagulation is the treatment of choice for cerebral venous thrombosis, the duration of treatment is another matter of debate. It also remains unclear what parameters should be used to evaluate the time period over which treatment is necessary. One would assume that recanalisation should play an important role in any decision about discontinuing oral anticoagulation, but no study has yet shown a clear cut association between recanalisation and clinical outcome.

Outcome at 12 months was good, as only a mild residual deficit was observed in $18 \%$ of the sample, and full recovery occurred in $82 \%$. This is in accordance with or slightly better than the outcome in previous studies. The relatively small sample size in the present study, together with the generally good outcome, prevented us from evaluating the influence of recanalisation on the clinical course.

Marked differences in recanalisation rate were evident depending on the site of venous thrombosis. The highest recanalisation rates were noted in the superior sagittal sinus $(94 \%)$ and the straight sinus ( $80 \%)$, while corresponding rates in the transverse sinus and the sigmoid sinus were significantly lower ( $57 \%$ and $41 \%$, respectively). To the best of our knowledge, recanalisation rates have not been systematically examined in any previous studies. Although this result is intriguing, various statistical problems reduce its validity: first, the small number of patients examined; second, correction for additional risk factors was not feasible, as more than one cerebral vein was affected in most patients; and third, it remains unclear how the coexistence of multiple thrombosed cerebral veins affects recanalisation-for example, whether an isolated thrombosis of a transverse sinus has the same chance of recanalising as a thrombosis of the transverse sinus associated with thromboses of the ipsilateral sigmoid sinus and the superior sagittal sinus.

Cross sectional TOF MRV was chosen for evaluation of cerebral venous recanalisation. This represents the standard imaging technique for cerebral venous thrombosis, with a good correlation with digital subtraction angiography. ${ }^{11}$ Cross sectional TOF MRV is quite sensitive to slow flow, particularly

Table 2 Site of sinovenous thrombosis at presentation and four and 12 months later in 33 patients with cerebral venous thrombosis

\begin{tabular}{|c|c|c|c|c|c|c|c|c|c|}
\hline & \multicolumn{2}{|c|}{ At presentation (n (\%)) } & \multicolumn{2}{|c|}{ At 4 months (n (\%)) } & \multicolumn{2}{|c|}{ At 12 months (n (\%)) } & \multicolumn{3}{|l|}{$\mathrm{p}$ Value } \\
\hline & Patients & Vessels & Patients & Vessels & Patients & Vessels & $\begin{array}{l}\text { Presentation } \\
\vee 4 \text { months }\end{array}$ & $\begin{array}{l}\text { Presentation } \\
\text { v } 12 \text { months }\end{array}$ & $\begin{array}{l}4 \text { months } v \\
12 \text { months }\end{array}$ \\
\hline Superior sagittal sinus & $16(48)$ & $16(48)$ & $1(3)$ & $1(3)$ & $1(3)$ & $1(3)$ & $<0.001$ & $<0.001$ & NS \\
\hline Straight sinus & $10(30)$ & $10(30)$ & $2(6)$ & $2(6)$ & $2(6)$ & $2(6)$ & $<0.01$ & $<0.01$ & NS \\
\hline Transverse sinus & $28(85)$ & $32(48)$ & $12(36)$ & $12(18)$ & $12(36)$ & $12(18)$ & $<0.0001$ & $<0.0001$ & NS \\
\hline Sigmoid sinus & $24(73)$ & $24(36)$ & $9(27)$ & $10(15)$ & $9(27)$ & $10(15)$ & $<0.001$ & $<0.001$ & NS \\
\hline Great cerebral vein & $3(9)$ & $3(9)$ & 0 & 0 & 0 & 0 & NS & NS & NS \\
\hline Internal cerebral vein & $3(9)$ & $5(8)$ & 0 & 0 & 0 & 0 & NS & NS & NS \\
\hline Jugular vein & $11(33)$ & 11 (17) & $3(9)$ & $4(6)$ & $3(9)$ & $4(6)$ & $<0.01$ & $<0.01$ & NS \\
\hline Cavernous sinus & $2(6)$ & $3(5)$ & 0 & 0 & 0 & 0 & NS & NS & NS \\
\hline
\end{tabular}


when thin slices are used, ${ }^{12}$ as was the case in this study. Obviously, we cannot exclude the possibility that very slow venous flow was misinterpreted as occlusion based on the MRV findings. However, as the magnetic resonance technique used represents the current standard and the performance of digital subtraction angiography would not be warranted or ethical, our methodology represents the best approach feasible.

Our results suggest that recanalisation only occurs within the first four months following cerebral venous thrombosis and not thereafter, irrespective of oral anticoagulation. These results could therefore provide some guidance on the necessary duration of oral anticoagulation following cerebral venous thrombosis, provided that recanalisation is accepted as a valid marker for this issue.

Our study has several limitations. First, the number of patients investigated was relatively small. However, cerebral venous thrombosis is a rare disease, and the two prospective, randomised, placebo controlled studies evaluating the efficacy of heparin in cerebral venous thrombosis have included only 20 and 60 patients, respectively. ${ }^{13}$ Second, no placebo group was used in the study because we did not consider the institution of a placebo arm to be ethical. Third, the duration of warfarin administration after the four months follow up was left to the discretion of the patient's physician. However, the length of warfarin treatment was similar in patients without and with recanalisation of their cerebral venous thromboses, suggesting that the warfarin did not affect the recanalisation rate.

\section{Conclusions}

Our data suggest that recanalisation of cerebral venous thrombosis in patients treated with warfarin is accomplished within the first four months. Late recanalisation was not observed in this study, irrespective of the use of oral anticoagulants.

\section{Authors' affiliations}

R W Baumgartner, A Studer, D Georgiadis, Department of Neurology, University Hospital of Zürich, Zürich, Switzerland M Arnold, Department of Neurology, University Hospital of Bern, Bern, Switzerland

Competing interests: none declared

\section{REFERENCES}

1 Einhäupl KM, Villringer A, Meister W, et al. Heparin treatment in sinus venous thrombosis. Lancet 1991;338:597-600.

2 Preter M, Tzourio C, Ameri A, et al. Long-term prognosis in cerebral venous thrombosis. Stroke 1996;27:243-6.

3 Bruijn de S, Stam J, for the Cerebral Venous Sinus Thrombosis Study Group. Randomised placebo-controlled trial of anticoagulant treatment with low-molecular-weight heparin for cerebral sinus thrombosis. Stroke 1999;30:484-8.

4 Mattle HP, Edelman RR, Reis MA, et al. Flow quantification in the superior sagittal sinus using magnetic resonance. Neurology 1990;40:813-15

5 Van Swieten JC, Koudstaal PJ, Vissr MC, et al. Interobserver agreement for the assessment of handicap in stroke patients. Stroke 1988;19:604-7.

6 Sachs L. Angewandte Statistik. Berlin: Springer, 1978:278

7 Charbonnier BA, Fiessinger J-N, Banga J-D, et al, on behalf of the FRAXODI group. Comparison of a once daily with a twice daily subcutaneous low molecular weight heparin regimen in the treatment of deep vein thrombosis. Thromb Haemost 1998;79:897-901.

8 Koopman MMW, Prandoni P, Piovella F, et al, for the Tasman Study Group. Treatment of venous thrombosis with intravenous unfractionated heparin administered in the hospital as compared with subcutaneous low-molecular-weight heparin administered at home. N Engl J Med 1996;334:682-7

9 Research Committee of the British Thoracic Society. Optimum duration of anticoagulation for deep-vein thrombosis and pulmonary embolism. Lancet 1992;340:873-6.

10 Schulman S, Rhedin A-S, Lindmarkker P, et al. A comparison of six weeks with six months of oral anticoagulant therapy after a first episode of venous thromboembolism. N Engl J Med 1995;332:1661-5.

11 Mattle HP, Wentz KU, Edelman RR, et al. Cerebral venography with MR. Radiology 1991;178:453-8.

12 Turski PA. Basic principles of MR angiography and flow analysis: cerebrovascular applications. In: Edelman RR, Hesselink JR, Zlatkin MB, eds. Clinical magnetic resonance imaging. Philadelphia: WB Saunders Co 1996:787-827. 